\title{
Systematic Series Expansions for Processes on Networks
}

\author{
M. B. Hastings ${ }^{1}$ \\ 1 Center for Nonlinear Studies and Theoretical Division, \\ Los Alamos National Laboratory, Los Alamos, NM 87545, hastings@cnls.lanl.gov
}

(Dated: October 21, 2005)

\begin{abstract}
We use series expansions to study dynamics of equilibrium and non-equilibrium systems on networks. This analytical method enables us to include detailed non-universal effects of the network structure. We show that even low order calculations produce results which compare accurately to numerical simulation, while the results can be systematically improved. We show that certain commonly accepted analytical results for the critical point on networks with a broad degree distribution need to be modified in certain cases due to disassortativity; the present method is able to take into account the assortativity at sufficiently high order, while previous results correspond to leading and second order approximations in this method. Finally, we apply this method to real-world data.
\end{abstract}

Historically, high temperature series expansions played an important role in the development of the theory of critical phenomena on regular lattices [1], showing nonclassical critical behavior and giving the first hints at the concept of universality. The success of these methods is remarkable when one considers that accurate results near the critical temperature require taking into account critical fluctuations on all scales. Such results were obtained by the ratio method, Pade approximants 2], and other means of extrapolating a series of finite length.

In this Letter, we apply series expansions to systems on networks. One expects the results to be more accurate at a given order on network systems than on regular lattices because in many cases these critical correlations are absent. On scale-free lattices there are no critical correlations due to the absence of a spatial structure 3 . On a small-world network [4], there are correlations determined by the regular lattice up to a certain scale and then mean-field correlations beyond. In the limit of a small density of long-range links on the small-world lattice, the correlations on the regular lattice lead to an anomalous dependence of the mean-field amplitudes on the link density [5]. For a large density of links, the meanfield behavior takes effect beyond a short scale, and thus we expect even a few terms of the high temperature series to suffice for accuracy.

Further, the series expansion method includes nonuniversal effects including details of the lattice structure. We will mostly focus on locating the critical point where we see that the series expansion method provides quantitatively accurate results that can be systematically improved by going to higher orders. Historically, series expansion methods have provided some of the most accurate results for critical exponents [1], so on this much simpler problem we expect that high temperature series can be more accurate that direct Monte Carlo simulation.

We begin by developing the series expansions for equilibrium problems, focusing on an Ising model as a definite example, and then develop the series expansion for non-equilibrium problems. We will see that many of the commonly accepted results for the critical point appear in the leading orders of the series expansion, while higher orders can significantly change the location of the critical point due to disassortativity in certain networks [7].

We are aware of one previous use of series for network problems 8 , for bond percolation, equivalent to certain models of epidemic disease. In this case, an exact relation was developed between the susceptibility of a regular lattice and the critical point of a small world network, and the susceptibility was determined using series expansion. We show that for a class of problems which we term "factorizable" similar exact relations can be found on small world networks. For example, we exactly locate the critical point in the Ising model on a one-dimensional smallworld network. The final result in this Letter is to show that the series expansion can be applied directly to an arbitrary real-world network with accurate results.

Equilibrium Problems - We consider an Ising model on a network as a typical equilibrium problem. We set all bonds to the same strength for simplicity to study the effect of network structure. We leave the network structure completely arbitrary here, and later give the results in certain special cases of the network structure.

The partition function is

$$
Z=\sum_{\left\{\sigma_{i}\right\}} \exp \left[J / k T \sum_{<i, j>} \sigma_{i} \sigma_{j}\right]
$$

where the sum is over nearest neighbors $i, j$ on the given network. We locate the critical point by calculating a high temperature series for the susceptibility, defined by $\chi=(k T)^{-1} N^{-1} \sum_{i, j}\left\langle\sigma_{i} \sigma_{j}\right\rangle$ where $N$ is the total number of sites. The susceptibility series is well-suited for locating the critical point because it diverges as $\left(T-T_{c}\right)^{-1}[\underline{3}]$ in these models, while the specific heat shows a jump at $T_{c}$ but gives no divergence above $T_{c}$.

Following standard techniques of high temperature series expansions [9], we calculate $\chi$ to fourth order:

$$
\begin{aligned}
& k T \chi=1+w \overline{n_{1}}+w^{2} \overline{n_{2}}+ \\
& w^{3} \overline{n_{3}}+w^{4}\left(\overline{n_{4}}-\overline{n_{\triangle}}\right)+\ldots,
\end{aligned}
$$

where $w=\tanh (J / k T)$ and where the numbers $\overline{n_{m}}$ characterize the network by giving the average over all sites 
$i$ of the number of self-avoiding paths of length $m$ connected to site $i$. Thus, $\overline{n_{1}}=\bar{k} \equiv N^{-1} \sum_{i} k_{i}$, where $k_{i}$ is the connectivity of a site $i$. One may see that $\overline{n_{2}}=$ $\overline{k^{2}-k} \equiv N^{-1} \sum_{i}\left(k_{i}^{2}-k_{i}\right)$. The number $n_{3}$ is equal to $N^{-1} \sum_{i, j, k ;<i, j>,<j, k>, i \neq k} 1$, the sum over triplets $i, j, k$ such that $i \neq k$ and $i, j$ and $j, k$ are pairs of nearest neighbors. We define $\overline{n_{\triangle}}=N^{-1} \sum_{i, j, k ;<i, j>,<j, k>,<i, k>} 1$, the average number of length-3 closed loops. Thus, for a plane triangular lattice, $n_{\triangle}=12$.

The simplest method of extracting the critical temperature is the ratio method: given a series $\sum_{n} a_{n} w^{n}$ given by Eq. (2), we approximate the radius of convergence, and hence the critical $w$, by by $a_{n} / a_{n-1}$. The first ratio, $a_{1} / a_{0}=\bar{k}$, giving $w^{-1}=\bar{k}$, and for $\bar{k}$ large this reduces to $k T_{c} \approx J \bar{k}$. The second ratio, $a_{2} / a_{1}$, gives $w^{-1}=\overline{k^{2}-k} / \bar{k}$. This second result is the commonly claimed result 3] for the critical point, improving on the naive result $\bar{k}$. We will see, however, that even higher orders in the ratios significantly modify this result by including effects of assortativity.

A better method of extracting the critical temperature is by means of Pade approximants [2]. We write a series with a finite number of terms as $\sum_{n=0}^{L+M} a_{n} w^{n}=$ $\left[\sum_{i=0}^{L} p_{i} w^{i}\right] /\left[\sum_{j=0}^{M} q_{j} w^{j}\right]+\mathcal{O} w^{L+M+1}$. The above series with four terms can be approximated by an $L=2$, $M=2$ Pade approximant. The closest singularity is at $w^{-1}=\frac{-a_{2} a_{3}+a_{1} a_{4}+\sqrt{\left(a_{2} a_{3}-a_{1} a_{4}\right)^{2}-4\left(a_{2} a_{4}-a_{3}^{2}\right)\left(a_{1} a_{3}-a_{2}^{2}\right)}}{2\left(a_{2} a_{4}-a_{3}^{2}\right)}$.

Illustration - We now apply these results to a simple class of networks with a broad, but finite, degree distribution and with nontrivial assortativity. We designate each node with probability $p$ as a "high degree" node, and with probability $1-p$ as a "low degree" node. For each high degree node, we assign $m$ connections from that node to other, randomly chosen, nodes. If two nodes become connected more than once via this procedure, we give them only a single connection. For $N$ large, a low degree nodes has a Poisson distribution of links, with average degree $p m$ and a high degree node has $k$ links where $k-m$ is chosen from a Poisson distribution with average $p m$. Thus, $\overline{n_{1}}=2 p m, \overline{n_{2}}=\overline{k^{2}-k}=$ $3 p^{2} m^{2}+p\left(m^{2}-m\right), \overline{n_{3}}=4 p^{3} m^{3}+4 p^{2}\left(m^{2}-m\right)(m)$, and $\overline{n_{4}}=5 p^{4} m^{4}+10 p^{3}\left(m^{2}-m\right) m^{2}+p^{2}\left(m^{2}-m\right)^{2}$.

For $p$ small and $m$ large the degree distribution becomes very broad and $\overline{n_{1}} \approx 2 p m, \overline{n_{2}} \approx p m^{2}, \overline{n_{3}}=$ $4 p^{2} m^{3}, \overline{n_{4}}=p^{2} m^{4}$. Thus, the ratio method for the critical point yields a series of approximations: $a_{1} / a_{0} \approx$ $2 p m, a_{2} / a_{1} \approx m / 2, a_{3} / a_{2} \approx 4 p m, a_{4} / a_{3} \approx m / 4$, and so on, with an obvious alternation from term to term. Using the Pade approximant, we find in the limit $p m>>$ $1, p<<1$ that

$$
k T_{c} \approx J \sqrt{\overline{k^{2}}} .
$$

This result (3) for $T_{c}$ is far below the second order ratio result, $k T_{c} \approx J \overline{k^{2}-k} / \bar{k}$ for the given limit. The

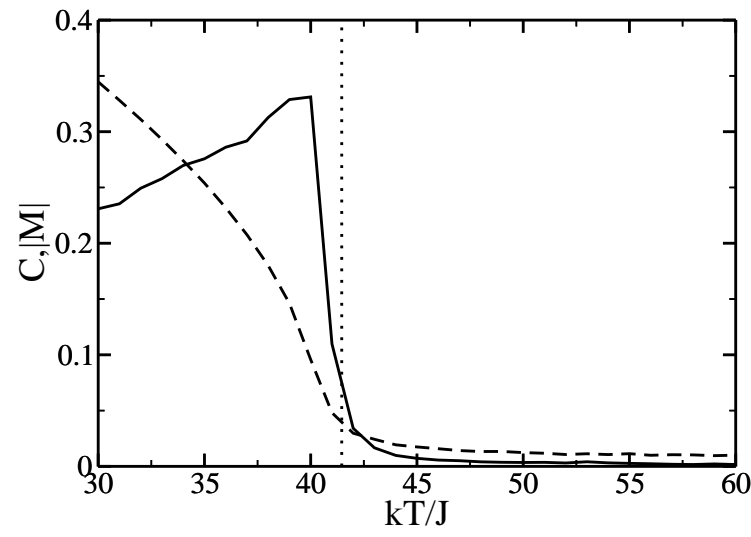

FIG. 1: Specific heat (solid line) and average of absolute value of magnetization (dashed line) for Monte Carlo simulation of 16384 spins. Pade approximant predicts critical point at $k T_{c} / J=41.46$, shown as vertical dotted line.

physical reason for this difference is the disassortativity of the given network. The high degree nodes connect to other nodes chosen at random, which are most likely to be low degree nodes. In the given limit, most of the paths of length $2 k$ consist of a path that alternates between high and low degree nodes, and thus there are of order $p^{k} m^{2 k}$ such paths, while there are only $p^{k} m^{2 k-1}$ paths of length $2 k-1$.

Following [7], we measure the assortativity of the network via the connected correlation function of remaining degrees at the end of a random edge. This quantity is equal to

$$
\left(\overline{n_{3}}+\overline{n_{\triangle}}\right) / \overline{n_{1}}-\left(\overline{n_{2}} / \overline{n_{1}}\right)^{2} .
$$

Thus, on a network with vanishing assortativity and no length three loops, the ratios $a_{3} / a_{2}$ and $a_{2} / a_{1}$ are equal. If the higher order ratios, $a_{n} / a_{n-1}$, are also equal then the critical $w^{-1}$ is correctly given by $\overline{n_{2}} / \overline{n_{1}}$.

In fact, the Pade approximant method is capable of yielding highly accurate numbers for specific systems even at this low order if the degree distribution is broad enough. We choose the specific case of $p=.1, m=100$ so that $\overline{n_{1}}=20, \overline{n_{2}}=1290, \overline{n_{3}}=43600, \overline{n_{4}}=2020100$. The ratio method gives the series of different ratios for the critical $w^{-1}: 20,64.5,33.8,46.33$, which show alternation from term to term but appear to be converging. The Pade approximant puts the critical temperature, $T_{c}=41.46$. We show in Fig. 1 curves for the specific heat and averge of the absolute value of the magnetization for a Monte Carlo simulation of 16384 spins on the given network. It can be seen that the Pade result is very close to the correct critical temperature, while the first and second ratio results $k T_{c} / J \approx 19.98$ and $k T_{c} / J \approx 64.49$ are poor estimates.

Exact Results in High Temperature Regime- In some cases, the high temperature series can be used to exactly locate the critical point. For the specific network 
discussed above, in the limit of large $N$ the loops in the network become very long and thus looplessness is a good approximation. One can show exactly that in this model $\overline{n_{k}} \sim\left(p m+\sqrt{p\left(m^{2}-m\right.}\right)^{k}$. In the Ising model, if the network is loopless, then the susceptibility series becomes $k T \chi=\sum_{k} w^{k} \overline{n_{k}}$, and thus the critical point is located at $w-1 \sim \lim _{k \rightarrow \infty}{\overline{n_{k}}}^{1 / k}$. In fact, this result precisely agrees with the Pade result above, so that the critical point located above is exact.

A second class of problems for which one can find exact results for the critical point consists of certain "factorizable" models on small-world networks 4 constructed according to the following procedure: one starts with a regular lattice in $d$-dimensions, and then adds long-range links, connecting any given pair of nodes with probability $p / N$. Consider the high temperature series expansion of an Ising model on such a network. At any fixed, finite order of the expansion for $k T \chi$, the only loops which appear in the limit of large $N$, are due to loops on the local lattice [10], with no loops due to shortcuts. Then, the ensemble average of the susceptibility in the high temperature regime on the small-world network in the large $N$ limit is exactly given by

$$
k T \chi=k T \chi_{0}(T) /\left[1-p \tanh (J / k T) k T \chi_{0}(T)\right],
$$

where $\chi_{0}(T)$ is the susceptibility at temperature $T$ on the local lattice with no shortcuts added. This is the same kind of result found in [8], and such results can be found for percolation, Ising, and Potts models, as well as the SIR model, but not the contact process or Heisenberg model where the series expansions can involve multiple crossing of the same small-world link. Similarly, models such as diffusion [ 6$]$ on a small-world network again do not have such an exact result because the model of diffusion in the absence of long-range links does not have a meaningful series expansion due to the presence of an infinite correlation length.

Eq. (5) accords with the mean-field theory result [5]. It locates the critical temperature of the one-dimensional small-world Ising model exactly: $p \tanh (J / k T) \exp [2 J / k T]=1$, so that for small $p$, $k T=2 J / \log \left(p^{-1}\right)+\ldots$.

Non-Equilibrium Problems - We now consider nonequilibrium processes on networks. The power series for these processes can be calculated just as for equilibrium processes, although the combinatorics are slightly more difficult. We consider the contact process 11] and SIR model [12]. In the contact process, sites are labeled either susceptible or infected, and an infected site can infect susceptible neighbors at rate $\lambda$, while it can recover and become susceptible with unit rate. In the SIR model, the infected sites become removed, a third state. Once a site reaches the removed state, that node can no longer become infected or infect its neighbors.

In the contact process, we start at time $t=0$ in a state with all sites susceptible and one site infected and then compute

$$
\chi_{S I S} \equiv \int_{0}^{\infty} \mathrm{d} t \sum_{i} n_{i}(t),
$$

where $n_{i}$ is the probability that site $i$ is infected at time $t$, averaged over all possible different initially infected sites. This quantity $\chi_{S I S}$ diverges at the epidemic threshold. One finds to second order

$$
\chi_{S I S}=1+\lambda \overline{n_{1}}+\lambda^{2} \overline{n_{2}}+\ldots
$$

For the SIR model, at $t=\infty$ all nodes are either susceptible or removed with no nodes susceptible. We define in this case $\chi_{S I R}$ to be the average number of removed nodes at $t=\infty$ averaged over the same set of initial state, giving

$$
\chi_{S I R}=1+\lambda \overline{n_{1}}+\lambda^{2}\left(\overline{n_{2}}-\overline{n_{1}}\right)+\ldots
$$

Finally, we consider bond percolation, which is related to, but not identical to, the SIR model, and in fact has a different series expansion. Bonds are active with probability $p$ and we define $\chi_{\text {perc }}$ to be the average number of sites connected to a given starting site. Here

$$
\begin{array}{r}
\chi_{\text {perc }}=1+p \overline{n_{1}}+p^{2} \overline{n_{2}}+p^{3}\left(\overline{n_{3}}-\overline{n_{\triangle}}\right)+ \\
p^{4}\left(\overline{n_{4}}-(3 / 2) \overline{n_{\square}}-2 \overline{n_{\triangle, 1}}\right)+\ldots,
\end{array}
$$

where $\overline{n_{\square}}=N^{-1} \sum_{i, j, k, l ;<i, j>,<j, k>,<k, l>,<l, i>, i \neq k, j \neq l} 1$, the average number of length- 4 closed loops, and where $\overline{n_{\triangle, 1}}=N^{-1} \sum_{i, j, k, l ;<i, j>,<j, k>,<k, l>,<l, j>, i \neq k, i \neq l} 1$. Thus, for a plane square lattice, $n_{\square}=8$.

At higher orders, the detailed series for these three different processes show differences, but the dominant term will be of the form $\lambda^{k}\left(\overline{n_{k}}+\ldots\right)$, so that if the network is highly connected with $n_{k}>>n_{k-1}$, we can locate the critical point at $\lambda \approx \lim _{k \rightarrow \infty}\left(n_{k-1} / n_{k}\right)$. Thus, the same results as in the Ising model case for the effects of assortativity occur again here.

Epidemics on Real-World Networks - We now consider the dynamics on real-world networks. We consider bond percolation as a version of the SIR process on the 283 node connected component of the web of romantic relationships at "Jefferson High" 13. Shown in Fig. 2 is the results of simulation as well as the Pade approximant results. We include the $[0,1]$ Pade approximant, $\chi_{\text {perc }} \approx 1 /\left(1-p \overline{n_{1}}\right)$, as well as the $[1,1]$ approximant, $\chi_{\text {perc }} \approx\left[1+\left(\overline{n_{1}}-\overline{n_{2}} / \overline{n_{1}}\right) p\right] /\left[1-\left(\overline{n_{2}} / \overline{n_{1}}\right) p\right]$, as well as the $[2,2]$ approximant to the fourth order series (9). The $[0,1]$ and $[1,1]$ results give the standard results for the critical point, while the $[2,2]$ approximant is seen to be much more accurate.

We believe that the difference between the $[2,2]$ approximant and the simulation is in fact due to finite size effects. Differences set in only when a sizable fraction of the network is on average part of the percolation cluster. 


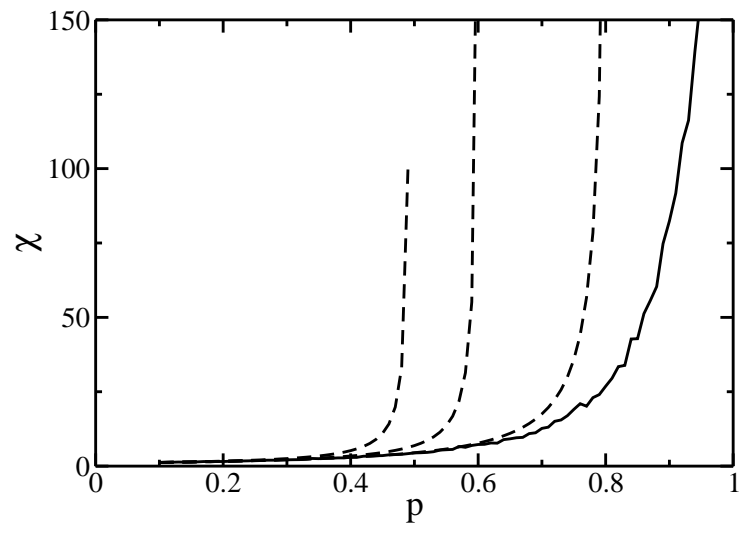

FIG. 2: Susceptibility of bond percolation on social network. Solid line is from simulation. Dashed lines are (from left to right) $[0,1],[1,1],[2,2]$ Pade approximant results.

We believe that if larger social networks were obtained, of the order of the number of people in a typical city, the $[2,2]$ approximant would become highly accurate. However, it is difficult to get large data on accurate social networks to test the series technique against simulation. In fact, this is precisely the reason for using the series technique: it allows one to obtain accurate results from local data.

Thus, to illustrate finite size effects, we return to the network defined in "Illustration", taking $p=.2$ and $m=10$. We find the results shown in Fig 3 . The $[2,2]$ approximant is again found to be more accurate, and it is seen that the simulation of 10000 sites is closer to the Pade result than the simulation of 1000 sites. As before, in fact, the Pade predicts the exact result for the critical point on this network in the infinite size limit.

The Pade result above can be determined for any network such that we can find the various constant $n_{1}, n_{2}, \ldots$. This can be determined based on local information and Monte Carlo sampling of a small portion of the network.

Discussion - The series expansion technique provides a systematic method for studying networks in the "high-temperature" phase. It is possible to extend this technique to include the ordered phase. For example, in an Ising model one can compute the partition function as $Z=\left\langle\exp \left[-J / k T \sum_{<i, j>} \sigma_{i} \sigma_{j}-\right.\right.$ $\left.\left.\sum_{i} h_{i} \sigma_{i}\right]\right\rangle_{h}=\exp \left[\sum_{n}(1 / n !)\left\langle\left(-J / k T \sum_{<i, j\rangle} \sigma_{i} \sigma_{j}-\right.\right.\right.$ $\left.\left.\left.\sum_{i} h_{i} \sigma_{i}\right)^{n}\right\rangle_{h, c}\right]$, where the expectation value $\langle\ldots\rangle_{h}$ is with respect to $\exp \left[+\sum_{i} h_{i} \sigma_{i}\right]$, and $\langle\ldots\rangle_{h, c}$ denote various cumulants. Keeping only the first cumulant, setting $h_{i}=h\left(k_{i}\right)$ for some function $h$ depending only on the local coordination number, and maximizing $Z$ over $h$ reproduces previous results $[\underline{3}$, while corrections are found at higher order.

The technique provides exact results on certain systems, such as loopless networks and certain problems on small-world networks, where it agrees with, but goes beyond, previous mean-field calculations. It works well on

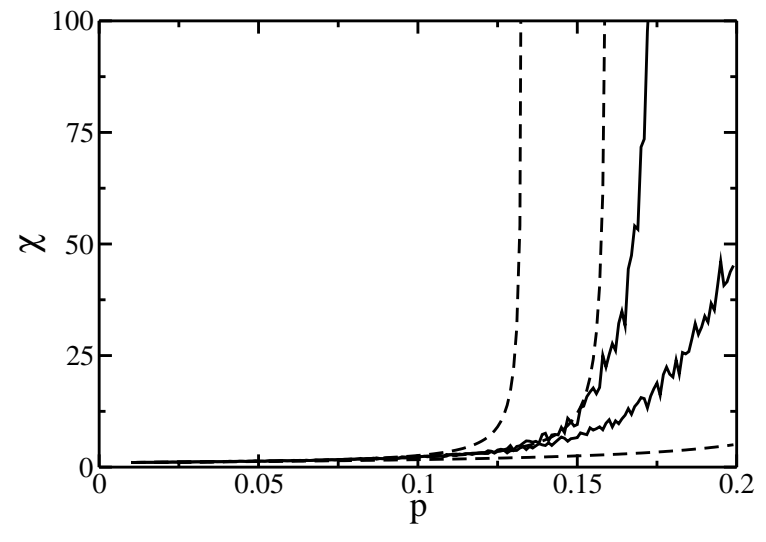

FIG. 3: Susceptibility of bond percolation on simulated network. Solid lines are (from left to right) from simulation of 10000 and 1000 nodes. Dashed lines are (from left to right) $[1,1],[2,2],[0,1]$ Pade approximant results.

simulated and realistic networks. In many cases, only local information on a network is available. A tendency has been then to construct a model which agrees with the local information, such as distribution of connectivities, and then simulate the desired process on such a simulated network. Series expansions instead directly express the desired result in terms of the local properties. One key result is the quantitative measure of how the assortativity affects the critical properties.

Acknowledgements - I thank M. A. Moore for useful discussions on the application of series expansions to critical phenomena. This work was supported by DOE grant W-7405-ENG-36.

[1] C. Domb, The Critical Point (Taylor \& Francis, Bristol, 1996), p. 148.

[2] G. A. Baker, Phys. Rev. 124, 768 (1961).

[3] A. V. Goltsev, S. N. Dorogovtsev, and J. F. F. Mendes, Phys. Rev. E 67, 026123 (2003); M. Leone, A. Vazquez, A. Vespignani, and R. Zecchina, Eur. Phys. J. B 28, 191 (2002).

[4] D. J. Watts and S. H. Strogatz, Nature 393, 440 (1998)

[5] M. B. Hastings, Phys. Rev. Lett. 91, 098701 (2003).

[6] B. Kozma, M. B. Hastings, and G. Korniss, Phys. Rev. Lett. 92, 108701 (2004).

[7] M. E. J. Newman, Phys. Rev. Lett. 89, 208701 (2002).

[8] M. E. J. Newman, I. Jensen, and R. M. Ziff, Phys. Rev. E 65, 021904 (2002)

[9] D. Jasnow and M. Wortis, Phys. Rev. 176, 739 (1968).

[10] C. Moore and M. E. J. Newman, Phys. Rev. E 62, 7059 (2000).

[11] J. Marro and R. Dickman, Nonequilibrium Phase Transitions in Lattice Models, (Cambridge University Press, 1999).

[12] P. Grassberger, Math. Biosci. 63, 157 (1983).

[13] H. Bruckner, A. Martin, and P. S. Bearman, Persp. on Sexual and Repr. Health. 36 (6), 248 (2004). 